\section{Diagnosis of irritable bowel syndrome would be better made by gastroenterologists than primary care physicians}

\author{
Mariette Bengtsson,' Bodil Ohlsson ${ }^{2}$ \\ 'Department of Nursing, Faculty of \\ Health and Society, Malmö University; \\ ${ }^{2}$ Department of Clinical Sciences, Division \\ of Gastroenterology and Hepatology, \\ Skåne University Hospital, Malmö, Lund \\ University, Sweden
}

\section{Abstract}

Irritable bowel syndrome (IBS) is a common disease, and constitutes a large portion of patients admitted to gastroenterology units. We wanted to examine whether there is a need for patients with suspected IBS to have a thorough examination by a gastroenterologist to establish the diagnosis, or whether other specialist or subspecialist physicians could better or equally identify the problems. From April 2003 to April 2005, females admitted with presumed IBS and consequently scrutinized by a gastroenterologist in our department were included. They were examined by a physician to establish a diagnosis. Four years later, the medical records were again scrutinized including abdominal symptoms, laboratory analyses and X-ray findings, to check if the findings were identical to the original diagnoses. Fifty admissions were identified. Nine of the patients did not want to participate, and 2 patients did not keep the appointment. The diagnosis of IBS was confirmed in only 20 (51\%) and the other 19 (49\%) had another diagnosis other than IBS. At follow up, 3 patients included with the IBS diagnosis had organic diseases, and 4 with another diagnosis also had IBS. Thus, $46 \%$ of the examined women with expected IBS had another diagnosis. A thorough examination of the patient and confirmation of the symptoms by a gastroenterologist is necessary before diagnosis of IBS is confirmed. For this purpose, patients need to meet a specialist when diagnosis is uncertain.

\section{Introduction}

Irritable bowel syndrome (IBS) is a common disease, and patients with this syndrome account for a large number of the patients seeking medical advice from gastroenterologists. ${ }^{1}$ The etiology for IBS is unknown, but hereditary and psychosocial factors, food in- tolerance and inflammation/infection have been discussed in this connection. ${ }^{2}$ Many of these patients seek medical attention and advice for many years at different levels. The treatment today is to alleviate symptoms. However, the sources of the patients' abdominal problems are often not simply medical, but also of a psychosocial character. ${ }^{3}$

We have in earlier studies shown how these patients have experienced inappropriate management by the medical care services, an uncertainty about diagnosis and an impaired quality of life compared to controls. Nurses are involved directly in the planning and implementation of therapeutic interventions for this patient population, and they can help patients with IBS by teaching them to help themselves. ${ }^{4.7}$ The question is whether it is necessary to refer all these patients to gastroenterologists, or whether primary care physicians and other subspecialist physicians could make a fair judgment of the patients' health problems and offer care management.

The aim of this study was to test a rational strategy for the investigation and management of IBS patients and, therefore, to evaluate whether there is a need to refer patients with suspected IBS to gastroenterologists.

\section{Materials and Methods}

\section{Patients}

All female patients with suspected IBS referred to the Department of Gastroenterology at the Skåne University Hospital, Malmö from April 2003 to April 2005 were consecutively examined by specialists in gastroenterology. They were Swedish-speaking women between 18-65 years old. They were re-assessed by the same specialist (B0) to evaluate whether all investigations performed were negative and whether the symptoms were in accordance with the Rome-II criteria for IBS. ${ }^{8}$ The women who fulfilled these inclusion criteria were asked to participate in the study. Men were not included in the study since men and women with IBS have different clinical pictures and a different perception of their quality of life. . $^{9-11}$ The patients identified were informed both in writing and by telephone by a specialist nurse in gastroenterology (MB), and they submitted their written informed consent before entering the study. The study was approved by the Ethics Committee at Lund University (LU 73502).

\section{Design}

After inclusion the patients received a questionnaire to complete before coming to the hospital for a medical assessment. The questionnaire included questions about previous
Correspondence: Bodil Ohlsson, Skåne University Hospital, Entrance 35, 20502 Malmö, Sweden. E-mail: bodil.ohlsson@med.lu.se

Key words: irritable bowel syndrome, irritable bowel syndrome, primary care physicians, diarrhea, constipation.

Acknowledgments: Mariette Bengtsson received financial support in the form of a study grant and postgraduate studentship from the Council of Health Research, Faculty of Medicine, Lund University. Bodil Ohlsson received financial support from the Development Foundation of Region Skåne, Skåne University Hospital, Malmö. We want to thank the colleagues at the Department of Gastroenterology, Skåne University Hospital, Malmö, for including patients in the study.

Contributions: $\mathrm{MB}$ and $\mathrm{B} 0$ together designed the study, collected the data, paid for the study and wrote the manuscript.

Conflict of interest: the authors report no conflicts of interest.

Received for publication: 17 April 2010.

Revision received: 21 June 2010.

Accepted for publication: 28 June 2010.

This work is licensed under a Creative Commons Attribution 3.0 License (by-nc 3.0)

(C) Copyright M. Bengtsson and B. Ohlsson, 2010 Licensee PAGEPress, Italy

Gastroenterology Insights 2010; 2:e10

doi:10.4081/gi.2010.e10

health and sickleave related to their gastrointestinal symptoms. In the Department of Gastroenterology, all patients met a physician for an ordinary medical assessment and possibly an additional investigation. The patients were also offered consultation with a dietician and a psychologist. The patients who visited the dietician completed a questionnaire at home about daily food intake to provide a picture of their eatingh abits before the visit. At the first visit, a diet anamnesis was assessed as a complement to the diet registration. The dietician gave basic dietary information and general advice on foods. At the second visit, 12 months later, the dietary advice given at the first visit was followed-up. The psychologist used the Beck Depression Inventory (BDI). ${ }^{12}$ This questionnaire contains 21 questions. Each question is graded from $0-3$ for a maximum of 63 points. The maximum score is categorized into 5 groups; normal below 10 , mild depression 10-15, mild to moderate depression 16-19, moderate to severe depression 20-29, severe depression above $29 .{ }^{13}$

Four years later, all medical records were reevaluated. Duration, frequency and/or intensity of symptoms of abdominal pain, cramp, dis- 
comfort, distension, bloating, change in stool habits, pain relief with defecation, stool frequency, stool characteristics, incomplete evacuation, straining and urgency were ascertained. Laboratory analyses X-ray studies were examined to reconsider the diagnosis.

\section{Statistical analyses}

Values for age were expressed as mean and standard deviation (SD) and for other values as the median and interquartile ranges (IQR). Differences between groups are calculated by Fisher's exact test, Kruskal-Wallis test and Mann-Whitney U test. $\mathrm{P}<0.05$ was considered statistical significance.

\section{Results}

Fifty patients fulfilled the inclusion criteria for the present study, but 9 of these decided not to participate. Of the remaining 41 who agreed to participate and were invited for a medical examination, 2 did not keep the appointment, resulting in 39 study patients who completed the study (Figure 1). Before inclusion in the study, the patients had visited the doctor an average two times during the previous year (interquartile range 1-3). The women had also visited other health practioners, such as a dietician, homeopathist, masseur, acupuncturist, and almoner. One of the women had visited a doctor 14 times. Two of the patients had been admitted to hospital for some days. The patients took few drugs for their gastrointestinal symptoms. At most, one patient took 3 different types of drugs.

The complete investigation of all patients included in the study was a time-consuming process. It took between 27 to 250 days from the time of admission until the patient had met a physician for the first time (median 94, IQR 64-153). The visit to the physician included a thorough medical examination. It took some time to complete the medical examination, since there was a delay for each X-ray examination (range 35-154 days). The patients went through the following examinations depending on symptoms: colonoscopy, enterography, capsule endoscopy, bowel transit time, and esophagogastroduodenoscopy, as well as examinations for fat and bile salt malabsorption and bacterial overgrowth, lymphocyte scintigraphy, and gastric emptying scintigraphy. At most, 2 patients went through 4 types of examinations. There was also a delay for the physician's follow-up contact, consultation, phone call or letter (Table 1). In general, the patients underwent a thorough examination in primary care before referral to the Department of Gastroenterology, and examinations were limited at the Department of
Gastroenterology. Of the included 39 patients with suspected IBS, only 20 patients had the diagnosis of IBS verified. Nine of these had diarrhea-predominated IBS (IBS-D), 6 constipated-predominated IBS (IBS-C) and 5 suffered from mixed IBS (IBS-M). At follow up four years later, 3 of those 20 patients had another diagnosis which could explain the gastrointestinal complaints that had first manifested some years earlier (Figure 1). One of the patients with IBS-D had suffered from IBS for 30 years when referred for the first time. The symptoms became worse in the last months before referral; a few months later, the patient had a severe weight loss and malignancy was suspected. Metastatic adenocarcinoma in the liver was confirmed with unknown origin, but cholangiocarcinoma was suspected. One of the patients with IBS-C suffered from abdominal bloating, gases and constipation. Further examination some years later revealed gastroparesis. When screening patients with capsule endoscopy at our department, one woman classified as IBS-M was shown to suffer from Cohn's disease as several ulcers and erosions were found in the small intestinal mucosa.

Nineteen of the patients were given a diagnosis other than IBS, but at follow up four years later, 4 of them were considered to suffer from IBS (Figure 1). The other 15, who were found not to have IBS, suffered from fat or bile salts malabsorption $(n=6)$, eating disturbances or drug abuse $(\mathrm{n}=3)$, bacterial overgrowth $(n=1)$, gastrointestinal reflux $(n=1)$, celiac disease $(n=1)$, collagenous colitis $(\mathrm{n}=1)$, Cohn's disease $(\mathrm{n}=1)$, Parkinson's disease $(n=1)$ and multiple severe diseases such

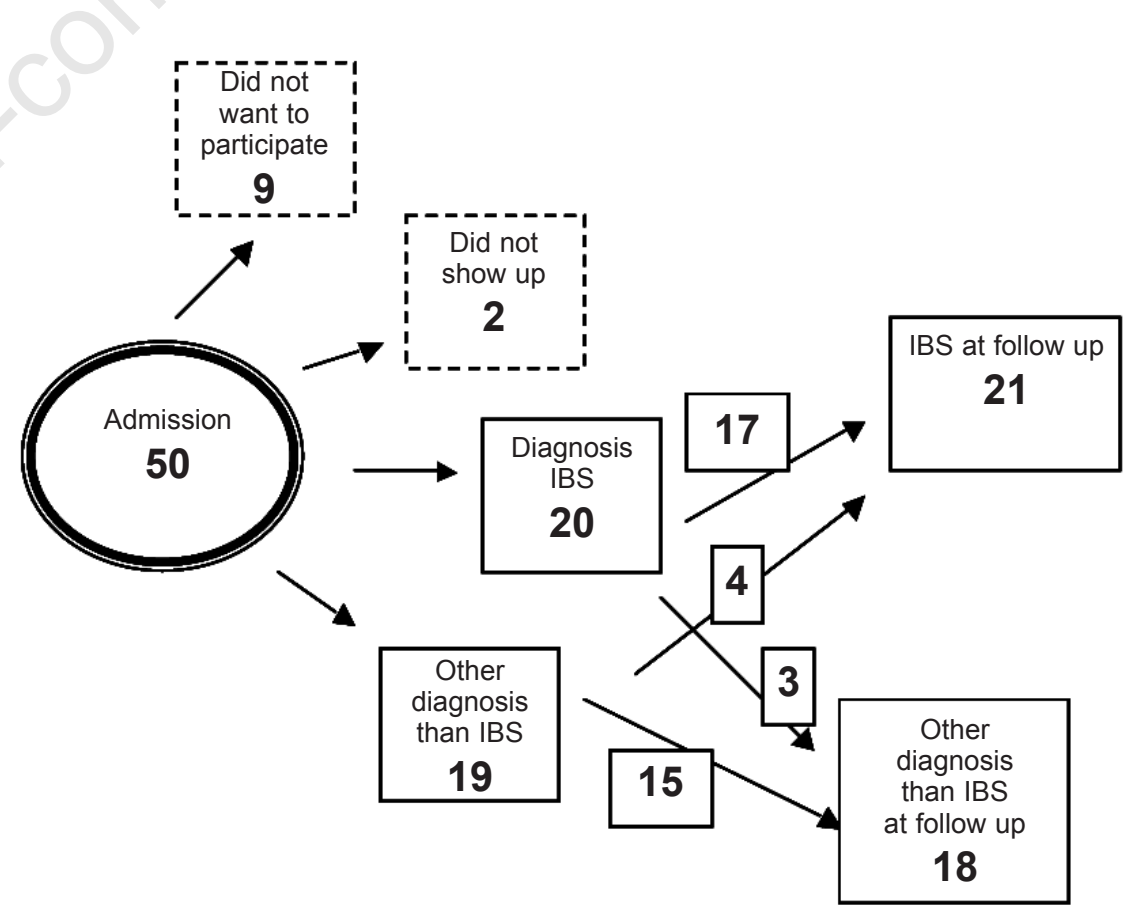

Figure 1. Flow-chart of the 50 patients who fulfilled the inclusion criteria for the study. as heart and renal failure $(n=1)$. Some of the patients had more than one diagnosis. Out of these, 7 suffered from alternating constipation and diarrhea, 6 from diarrhea and 2 from constipation. Four patients were initially diagnosed with lactose intolerance but also fulfilled the criteria for IBS at follow up as even though they had excluded lactose from their diet they still had symptoms. An overview of patients' characteristics is given in Tables 1 and 2.

Patients with IBS visited a dietician and psychologist more often, and used fewer pharmaceutical drugs than patients with other gastrointestinal diagnoses (Table 1). There was no statistically significant difference between the symptoms or signs in the patients who were diagnosed as having IBS and those who did not have this disorder. The prevalence of alarm symptoms in the form of weight reduction, bloody stool or nocturnal diarrhea was small in both groups. Pathological laboratory analyses seldom revealed anemia, low albumin levels in plasma and/or elevated C-reactive protein (CRP) (Table 2).

Analysis of the patients' eatingh abits showed that they almost all ate fruit daily but more rarely ate vegetables. All of them ate sweets at least once a day. According to the Nordic Nutrition Recommendations, intake of vitamin $\mathrm{C}$ and $\mathrm{D}$ was too low in all patients, and 6 of the 9 patients had too low an intake of dietary fiber, iron and calcium. ${ }^{14}$

In all, 6 patients were referred to a psychologist for therapy and 2 patients had already established a patient-physician relationship with a psychologist. Further information concerning these 2 patients' psychological state was not collected for the study due to the hos- 
pital security regulations. The psychologist considered that all 6 patients referred to them had benefited from psychotherapy. The patients gave similar details: an unhappy childhood during which they never felt that they were loved or that they were good enough and/or they had had to look after their younger brothers and sisters. Now, as adults they still have the responsibility for their own lives as well as for those of others, for example, their adult children, old parents and/or sick family members. The patients found it difficult to set limits to the demands other people made on them. The Beck Depression Inventory (BDI) was used as a complement to the psychologist's assessment of 4 of the 6 patients referred to them. ${ }^{12}$ Of these 4 , one was considered normal but according to the BDI the other 3 were depressed.

\section{Discussion}

The most important finding in the present study was that a thorough examination and evaluation of the patients with IBS symptoms must be performed by gastroenterologists. Although the doctor at the primary care center suspected IBS and performed basic examinations, and a specialist in Gastroenterology had scrutinized all admissions, about half of them received a diagnosis other than IBS after a personal meeting and examination by a specialist in Gastroenterology. Thus, a disease with organic or dysfunctional origin must be excluded and the IBS diagnosis must be thoroughly confirmed. ${ }^{15,16}$ Although nursing management of IBS patients has been reported to be adequate and efficient the diagnosis must first of all be confirmed before treatment is established for these patients. ${ }^{5,7}$

Although there many IBS patients referred to specialists, and they constitute about half of all patients, the most efficient strategy still seems to be to program one initial visit to a specialized physician for a final judgement and then personalize care. ${ }^{1}$ Clinically, specialists in gastroenterology have a tendency to reject patients admitted for suspected IBS at once, and they expect these patients to be handled at primary care centers. As the number of IBS patients is large, it is not possible for all patients to have regular contact with a specialist in Gastroenterology. However, early examination by a gastroenterologist may be necessary to confirm the diagnosis and confirm the patient's own perception concerning his or her illness before referral to a nurse or the primary care center for further management. ${ }^{17,18}$ It seems reasonable that all patients suffering from IBS-like symptoms should visit a gastroenterologist at least once if the diagnosis is in the least uncertain. Most patients not fulfilling the IBS criteria suffered from malabsorption diseases, and not from

Table 1. The number of contacts and pharmacological treatment.

\begin{tabular}{lccc} 
& $\begin{array}{l}\text { Patients with IBS } \\
\text { Patients without IBS }\end{array}$ & P \\
N. patients who met a & & & \\
$\quad$ Doctor & 21 & 18 & \\
Dietician & 12 & 2 & 0.01 \\
Psychologist & 6 & 0 & 0.02 \\
Physiotherapist & 2 & & NS \\
N. phone calls to a doctor/patient & 13 & NS \\
0 & 4 & 2 & \\
1 & 1 & 4 & \\
2 & 1 & 0 & \\
3 & 1 & 0 & \\
4 & 1 & 0 & NS \\
5 & 1 & $2(1-2)$ & NS \\
6 & $2(1-2)$ & $1(0-2)$ & \\
7 & $1(0-2)$ & & \\
Median n. visits to a doctor per patient & & & \\
Median n. phone calls to a doctor per patient & & 2 & \\
Pharmaceutical drugs related to & 12 & 6 & \\
gastrointestinal symptoms & 4 & 6 & \\
No drugs & 4 & 4 & \\
1 kind of drug & 1 & & \\
2 kinds of drugs & & & \\
3 kinds of drugs & & & \\
\hline
\end{tabular}

Values are given as median and interquartile ranges (IQR). Kruskal Wallis test or Mann-Whitney U test was used for statistical calculations.

Table 2. Patients' characteristics at referral for gastrointestinal complaints with the suspected diagnosis of irritable bowel syndrome.

$\begin{array}{lccc} & \text { Patients with IBS } & \begin{array}{c}\text { Patients without IBS } \\ \text { N P }\end{array} \\ \text { N. patients } & \text { N }(\%) & 18 & \\ \text { Mean age } \pm \text { SD at referral } & 21 & 40 \pm 15 & \text { NS } \\ \text { Smokers } & 34 \pm 13 & 6(27) & \text { NS } \\ \text { Gastrointestinal symptoms at referral } & 8(38) & & \\ \text { Diarrhea } & & 13(72) & \text { NS } \\ \text { Constipation } & 9(43) & 9(50) & \text { NS } \\ \text { Abdominal pain } & 8(38) & 17(94) & \text { NS } \\ \text { Abdominal bloating } & 21(100) & 9(50) & \text { NS } \\ \text { Abdominal gas } & 12(57) & 7(39) & \text { NS } \\ \text { Alarm symptoms } & 10(48) & 3(14) & \text { NS } \\ \text { Pathological laboratory data* } & 1(5) & 8(38) & \text { NS } \\ \text { Sick-leave due to gastrointestinal symptoms } & 3(14) & & \\ \text { 12 months before inclusion } & & 15(83) & \text { NS } \\ \text { No reported sick-leave } & 11(58) & 0 & \\ \text { Less than one week } & 2(8) & 0 & \\ \text { At least one week and less than one month } & 6(25) & 3(17) & \\ \text { More than one month } & 2(8) & \end{array}$

$\mathrm{N}(\%)=$ absolute number and $\%$ of total number of patients with IBS at the follow up four years later. Standard deviation=SD. All values except age are given as median (interquartile range (IQR)). * pathological laboratory data represented anemia, lowered albumin concentration in plasma and/or elevated C-reactive protein (CRP). Differences between groups were calculated by Fisher's exact test, Kruskal-Wallis test or Mann-Whitney U test.

diseases found by ordinary X-ray examinations. It is not possible for the primary care physicians to identify all these different conditions. There is often a discrepancy between patient's and physician's needs. Patients want to be managed at a specialist clinic because they feel they are really ill, but the specialist physician prefers the patients to be managed in primary care, as they do not suffer from a severe, organic disease demanding potential drug treatment.

Alarm symptoms in the form of weight reduction may be seen in patients with IBS as they avoid food because of accelerated abdominal pain after food intake. Some of the patients with IBS had slightly lowered albumin levels in plasma, possibly depending on insufficient or bad quality of food intake. They could have a transient high C-reactive protein (CRP), depending on a concomitant infection.

The intention of the study was that all referred patients should meet a nurse, dietician and a psychologist to make a professional multidisciplinary investigation to find out the true reason for the symptoms. We suspected 
that many of patients with IBS-like symptoms may have psychological disturbances and/or bad food intake. However, most patients with IBS did not agree to go to see a psychologist. Furthermore, as so many patients had diagnoses other than IBS, it was not thought relevant for them to see a dietician or psychologist. This partly explains the difference in visits to these professionals in Table 1. An examination by a nurse would be helpful to identify the problem, but a true diagnosis must be established before a nurse can plan care. ${ }^{19}$

There may have been a selection and referral bias in the present study since patients were referred from primary care. More patients with diarrhea than with constipation were referred. Patients with anxiety, depression or atypical symptoms are more often referred to a specialist for a second opinion than patients with ordinary symptoms and good coping mechanisms..$^{20,21}$ The optimal study would be to evaluate IBS-like symptoms in the primary care center to find out the prevalence of true IBS among these patients.

Studies comparing Manning, Rome I and Rome II criteria have shown different sensitivity in diagnosing IBS, with a low concordance rate between the three diagnostic criteria, illustrating the difficulties in diagnosing IBS. $^{22,23}$ A $100 \%$ specificity of criteria for IBS would be impossible as IBS-like symptoms are also common in organic diseases, but organic disease excludes IBS diagnosis. ${ }^{1}$ IBS-like symptoms are found in high prevalence in patients suffering from IBD. ${ }^{24}$ Laxative abuse, eating disturbances, lactose intolerance and neurological diseases may all give raise to IBSlike symptoms. ${ }^{25,26}$ These difficulties in diagnosing IBS correctly may be considered in studies trying to examine the etiology and pathophysiology of IBS.

Three of 4 patients (13\% of all included in the study) examined by the BDI were considered to suffer from a clinical depression, and 2 had a long history of psychological therapy. If all patients had been examined, perhaps more than these 3 would have been diagnosed as depressed. Many of the patients had insufficient nutritional intake. In an examination of a large number of students with IBS in another study, it was found that those not suffering from IBS spent more hours exercising and ate more regular meals than those with IBS. ${ }^{27}$ IBS sufferers are most probably a heterogeneous group of patients. Some of these may have their symptoms because of life-style factors, and may thus benefit from contact with, for example, a dietician. ${ }^{28}$ Physical activity and improvement of food intake has in other studies been shown to ameliorate the symptoms. ${ }^{29}$

In summary, the most important finding in this study is that a thorough examination and confirmation of the IBS diagnosis by a gastroenterologist is necessary before the diagno- sis is established. Eighteen of 39 patients included received a diagnosis other than IBS even though this had been suggested by the primary care physicians and was suspected by a specialist who had read the referral.

\section{References}

1. Thompson WG, Heaton KW, Smyth GT, Smyth C. Irritable bowel syndrome in general practice: prevalence, characteristics, and referral. Gut 2000;46:78-82.

2. Arebi N, Gurmany S, Bullas D, et al. Review article: the psychoneuroimmunology of irritable bowel syndrome--an exploration of interactions between psychological, neurological and immunological observations. Aliment Pharmacol Ther 2008;28:830-40.

3. Palsson OS, Drossman DA. Psychiatric and psychological dysfunction in irritable bowel syndrome and the role of psychological treatments. Gastroenterol Clin North Am 2005;34:281-303.

4. Bengtsson M, Ohlsson B. Psychological well-being and symptoms in women with chronic constipation treated with sodium picosulphate. Gastroenterol Nurs 2005;28:3-12.

5. Bengtsson M, Ulander K, Börgdal EB, et al. A course of instruction for women with irritable bowel syndrome. Patient Educ Couns 2006;62:118-25.

6 Bengtsson M, Ohlsson B, Ulander K. Women with Irritable Bowel Syndrome and their perception of a good quality of life. Gastroenterol Nurs 2007;30:74-82.

7. Heitkemper M, Levy RL, Jarrett M, Bond EF. Interventions for irritable bowel syndrome: a nursing model. Gastroenterol Nurs 1995;18:224-30.

8. Thompson WG, Longstreth GF, Drossman DA, et al. Functional bowel disorders and functional abdominal pain. Gut 1999;45: II43-7.

9. Corney RH, Stanton R. Physical symptom severity, psychological and social dysfunction in a series of outpatients with irritable bowel syndrome. J Psychosom Res 1990;34:483-91.

10. Dancey CP, Backhouse S. Towards a better understanding of patients with irritable bowel syndrome. J Adv Nurs 1993;18:144350.

11. Heitkemper M, Carter E, Ameen V, et al. Women with irritable bowel syndrome. Differences in patients' and physicians' perceptions. Gastroenterol Nurs 2002;25: 192-200.

12. Beck AT, Ward CH, Mendelson M, et al. Inventory for measuring depression. Arch Gen Psychiatry 1961;4:561-71.

13. Steer RA, Beck AT, Garrison B. Applications of the Beck Depression Inventory. In: Assessment of Depression. Edited by Sartorius N, Ban TA. Berlin: SpringerVerlag; 1986.

14. Becker W, Lyhne N, Pedersen AN, et al. Nordic Nutrition Recommendations 2004 Integrating nutrition and physical activity. Copenhagen: Nordic Council of Ministers; 2004:13.

15. Torii A, Toda G. Management of irritable bowel syndrome. Intern Med 2004;43:353-9.

16. Cash BD, Chey WD. Irritable bowel syndrome - an evidence-based approach to diagnosis. Aliment Pharmacol Ther 2004; 19:1235-45.

17. Shen B, Soffer E. The challenge of irritable bowel syndrome: creating an alliance between patient and physician. Cleve Clin J Med 2001;68:224-5, 229-33, 236-7.

18. Araldi 0, Barkin JS. Irritable bowel syndrome: update on pathogenesis and management. Med Princ Pract 2002;11:2-17.

19. Bengtsson M, Ulander K, Börgdal EB, Ohlsson B. A holistic approach for planning care of patients with irritable bowel syndrome. Gastroenterol Nurs 2010;33:98-108.

20. Levy RL, Olden KW, Naliboff BD, et al. Psychosocial Aspects of the Functional Gastrointestinal Disorders. Gastroenterol 2006;130:1447-58.

21. Spiller R, Aziz Q, Creed F, et al. Guidelines on the irritable bowel syndrome: mechanisms and practical management. Gut 2007; 56:1770-98.

22. Talley NJ, Phillips SF, Melton LJ, et al. Diagnostic value of the Manning criteria in irritable bowel syndrome. Gut 1990;31: 7781.

23. Yale SH, Musana AK, Kieke A, et al. Applying case definition criteria to irritable bowel syndrome. Clin Med Res 2008;6: 9-16.

24. Grover M, Drossman DA. Pain management in IBD. Inflamm Bowel Dis Monit 2009;10:1-10.

25. Boyd C, Abraham S, Kellow J. Psychological features are important predictors of functional gastrointestinal disorders in patients with eating disorders. Scand J Gastroenterol 2005;40:929-35.

26. Chiu CM, Wang CP, Sung WH, et al. Functional magnetic stimulation in constipation associated with Parkinson's disease. J Rehabil Med 2009;41:1085-9.

27. Kim YJ, Ban DJ. Prevalence of irritable bowel syndrome, influence of lifestyle factors and bowel habits in Korean college students. Int J Nurs Stud 2005;42:247-54.

28. Talley NJ. Irritable bowel syndrome. Practical management. Aust Fam Physician 2000;29:823-8.

29. Burden S. Dietary treatment of irritable bowel syndrome: current evidence and guidelines for future practice. J Hum Nutr Diet 2001;14:231-41. 